\title{
Detektion der Bindung von Biomolekülen an Biohybrid- Hydrogele mittels SPR und elektrokinetischen Messungen
}

\author{
Juliane Posseckardt ${ }^{1}$, Ralf Zimmermann ${ }^{2}$, Carsten Werner ${ }^{2}$ und Michael Mertig ${ }^{1,3}$ \\ ${ }^{1}$ Kurt-Schwabe-Institut für Mess- und Sensortechnik e.V. Meinsberg, Waldheim \\ posseckardt@ksi-meinsberg.de \\ ${ }^{2}$ Leibniz-Institut für Polymerforschung Dresden e.V., Dresden \\ ${ }^{3}$ Technische Universität Dresden, Dresden
}

\begin{abstract}
Kurzfassung:
Biohybrid-Hydrogele aus natürlichen und synthetischen Komponenten werden im Bereich der regenerativen Therapien zunehmend eingesetzt. Die weitere Entwicklung und Anwendung dieser Materialien erfordert neue Möglichkeiten für die In-situ-Detektion der Bindung und Freisetzung von Biomolekülen, z. B. Wachstumsfaktoren. Miniaturisierte Sensoren, die während der Bildungsphase direkt in die Hydrogele integriert werden, können hierzu einen entscheidenden Beitrag leisten. Hier werden erste Ergebnisse für die Erprobung der Oberflächenplasmonenresonanzspektroskopie (engl.: surface plasmon resonance, SPR) und der elektrokinetischen Messungen für die Detektion der Anbindung von Proteinen in Hydrogelen aus sternförmig-verzweigtem Polyethylenglycol (starPEG) und Heparin vorgestellt.
\end{abstract}

Schlüsselwörter: Biohybrid-Hydrogele, Heparin, star-PEG, SPR, elektrokinetische Messungen.

\section{Einleitung und Motivation}

Für die Entwicklung neuer regenerativer Therapienansätze werden zunehmend Biohybrid-Hydrogele aus synthetischen Komponenten und natürlichen Glycosaminoglycanen eingesetzt. Auf Grund der negativen Ladung der Glycosaminoglycane können diese Materialien Wachstumsfaktoren über elektrostatische Wechselwirkungen binden, diese über einen längeren Zeitraum freisetzen und damit Regenerationsprozesse fördern. Hierfür ist die genaue Kenntnis der Bindung der Faktoren in den Hydrogelen bzw. von deren Freisetzung aus den Hydrogelen notwendig. Die zur Ableitung der entsprechenden Materialkonstanten erforderlichen Untersuchungen sollen durch Oberflächenplasmonenresonanzspektroskopie (Kinetik, Beladung) und elektrokinetische Messungen zur Bestimmung der Grenzflächenleitfähigkeit (Beladung) realisiert werden.

\section{Materialien und Methoden}

Zusammensetzung der Biohybrid-Hydrogele

Biohybridgele aus aminofunktionalisiertem, sternförmig verzweigten Poly(ethylenglykol) (starPEG, MW 10.000, Polymer Source Inc., Kanada) und Heparin (MW 14.000, Cal- biochem, Deutschland) (Bild 1) wurden durch Vernetzung der Aminogruppen des starPEGs mit den Carboxygruppen des Heparins unter Nutzung von 1-Ethyl-3-(3-dimethylaminopropyl)carbodiimid (EDC, Sigma, Deutschland) und N-Hydroxysulfosuccinimid (sNHS, Sigma, Deutschland) hergestellt [1]. Das molare Verhältnis von starPEG zu Heparin wurde zur Variation der mechanischen Eigenschaften der Gele (Steifigkeit) variiert.

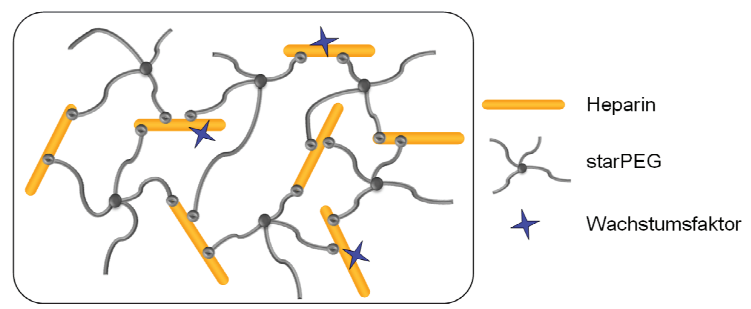

Bild 1: Zusammensetzung der verwendeten Hydrogele.

Beide Komponenten wurden in eisgekühltem $0,2 \mathrm{M}$ Phosphatpuffer $(\mathrm{pH} 7,4)$ gelöst. Nach der Zugabe des EDC und des sNHS zum Heparin wurde die Mischung zur Aktivierung der Carboxygruppen des Heparins auf Eis inkubiert und anschließend das gelöste starPEG zugegeben. Unmittelbar darauf erfolgte die Immobilisierung auf den Sensoroberflächen. 
(a)

(b)

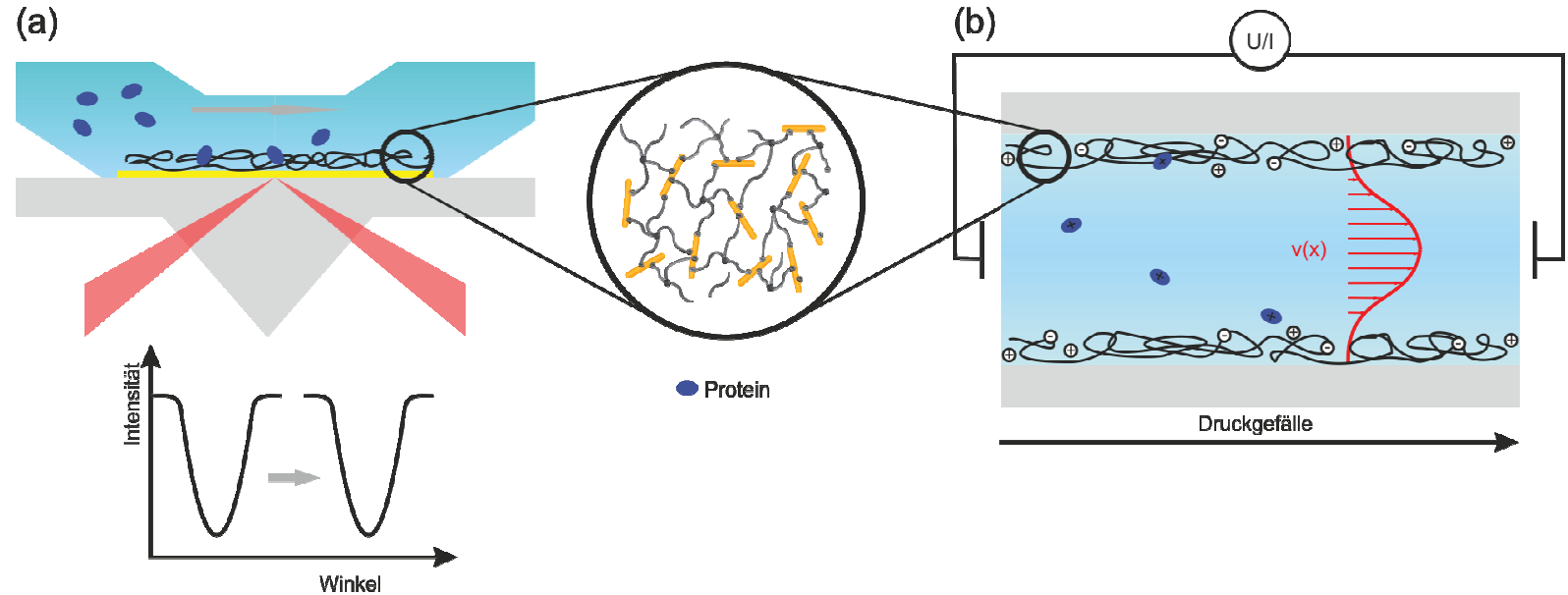

Bild 2: Schematische Darstellung des Messprinzips von (a) SPR und (b) Strömungsstrom- und Strömungspotentialmessungen zur Bestimmung der Grenzflächenleitfähigkeit.

\section{Immobilisierung auf den SPR-Chips}

Die Plasmonen-aktive Goldoberfläche der verwendeten SPR-Chips (capitalis $\mathrm{GmbH}$, Deutschland) wurde nach einer Ultraschallreinigung ( $5 \mathrm{~min}$, reinst $\mathrm{EtOH}$ ) durch Mikrokontaktdrucken von Octadecylmercaptan (Sigma, Deutschland) in hydrophobe und hydrophile Bereiche wie in [2] beschrieben strukturiert. Anschließend wurden die fertig gemischten aber noch nicht vernetzten Biohybrid-Hydrogele mit Hilfe eines Nanoplotters (GeSiM mbH, Deutschland) auf dem SPR-Chip immobilisiert. Da für die SPR-Messung eine linienförmige Immobilisierung der BiohybridHydrogele auf der Goldfläche notwendig ist, wurden zuerst Tropfen mit einem Abstand von $200 \mu \mathrm{m}$ abgesetzt. Anschließend wurde in alle Zwischenräume ein weiterer Tropfen dosiert (Bild 3a). Als Referenz wurde $100 \mathrm{mM}$ Poly(ethylenglykol)methyletherthiol (PEGthiol, Sigma, Deutschland) im 0,2 M Phosphatpuffer (pH 7,4) ebenfalls linienförmig immobilisiert. Nach der Vernetzung über Nacht wurden die Chips in 0,2 M Phosphatpuffer $(\mathrm{pH} \mathrm{7,4)} \mathrm{dreimal}$ für je eine Stunde gewaschen.

Immobilisierung auf den Sensoroberflächen für die elektrokinetische Messung

Zur Immobilisierung der Hydrogele auf den Substraten für die elektrokinetischen Messungen (thermisch oxidierte Siliziumwaferstücke) wurden diese mit einer dünnen Schicht aus Poly(ethylen-alt-maleinsäureanhydrid) funktionalisiert. Auf diese Schicht wurde die Mischung aus beiden Hydrogelkomponeten (siehe oben) pipettiert und mit einem Teflon ${ }^{\circledR}$ AF-beschichteten Deckgläschen abgedeckt. Nach der Vernetzung der Gelkomponenten wurde das Deckgläschen entfernt. Die Dicke der erhaltenen Hydrogelschichten lag im Bereich einiger Mikrometer.

\section{SPR-Spektroskopie}

Bei der SPR-Spektroskopie werden die Änderungen der dielektrischen Eigenschaften in einer ca. $200 \mathrm{~nm}$ dünnen Schicht oberhalb der ca. $50 \mathrm{~nm}$ dicken Goldschicht infolge von Bindungsereignissen detektiert (Bild 2). Als Messsignal wird die Verschiebung des Minimums des reflektierten Anregungsstrahls im Winkelspektrum („SPR-Signal“) verwendet.

\section{Elektrokinetische Messungen}

Die elektrokinetischen Phänomene Strömungspotential und Strömungsstrom basieren auf der Ausbildung einer elektrochemischen Doppelschicht an Fest-/Flüssig-Phasengrenzen [3]. Beide Größen wurden zur Bestimmung der Grenzflächenleitfähigkeit an einem von zwei Hydrogel-beschichteten Substraten gebildeten Mikrokanal in Abhängigkeit von Differenzdruck einer den Kanal durchströmenden Elektrolytlösung gemessen [4]. Die Grenzflächenleitfähigkeit reflektiert die Überschussladung im Gel und ändert sich durch die Anbindung von Proteinen über elektrostatische Wechselwirkungen.

\section{Ergebnisse}

In einer Reihe von Vorversuchen wurde die Immobilisierung der Hydrogele auf beiden Sensoroberflächen (linienförmige Beschichtung für SPR, Bild 3b, ganzflächige Beschichtung für elektrokinetische Messungen) optimiert. Es konnte gezeigt werden, dass die erhaltenen Hydrogelschichten unter Scherbelastung (in Folge der Durchströmung der Messzellen mit Puffer/Elektrolyt) delaminationsstabil sind.

\section{SPR-Messungen}

Referenzmessungen an Hydrogelen ohne Beladung mit Wachstumsfaktoren haben gezeigt, dass die Gele mit einem höheren 
(a)

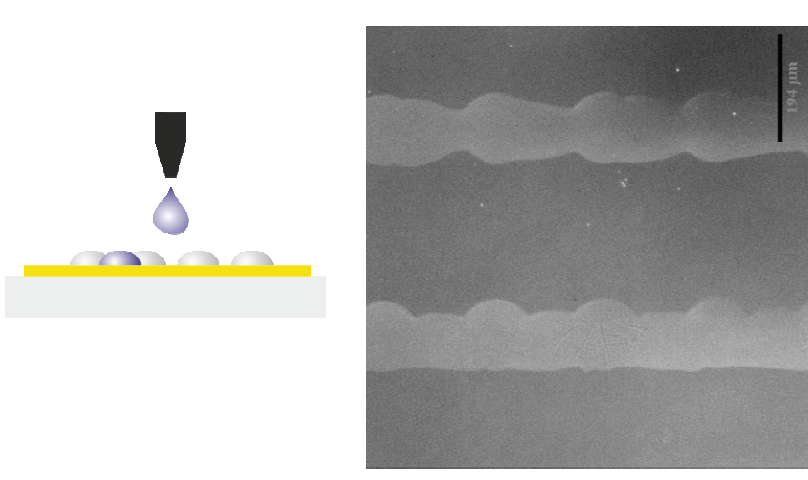

(c)

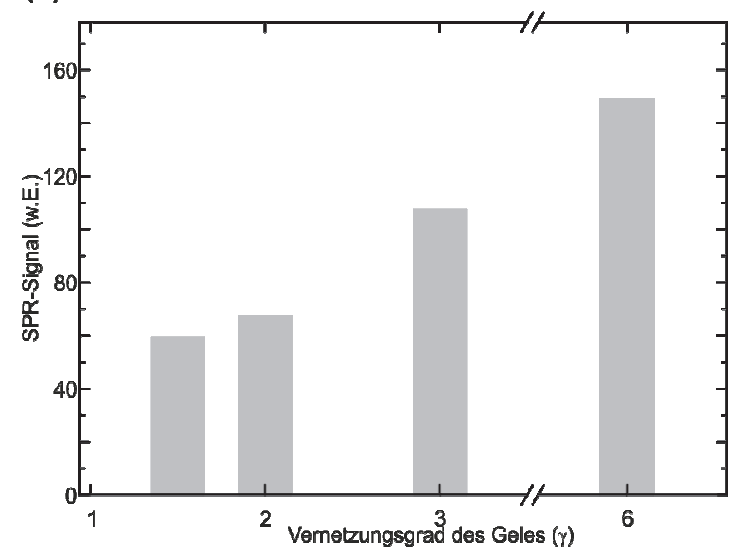

Bild 3: Immobilisierung der Hydrogele auf der Goldoberfläche des SPR-Chips. (a) Schematische Darstellung der linienförmigen Immobilisierung der Hydrogele auf den SPR-Chips. (b) Phasenkontrastaufnahme von unterschiedlichen Hydrogelen auf der Goldfläche des SPR-Chips. (c) Verschiebung der Minima des reflektierten Lichtes im SPR nach dem Immobilisieren der Gele.

Vernetzungsgrad $\gamma$ (molares Verhältnis starPEG zu Heparin) ein höheres SPR-Signal hervorrufen (Bild 3c). Dieser Befund korreliert mit der Zunahme der Netzwerkdichte/geringeren Quellung der Gele bei höherem Vernetzungsgrad. Anschließend konnte die Anbindung von Lysozym an das Hydrogel mittels SPR nachgewiesen werden (Bild 4). Lysozym hat eine vergleichbare Größe wie relevante Wachstumsfaktoren und einen im basischen $\mathrm{pH}$-Bereich liegenden isoelektrischen Punkt. An die Gele mit einem geringen Vernetzungsgrad konnte mehr Lysozym angebunden werden als an Gele mit einem höheren Vernetzungsgrad.

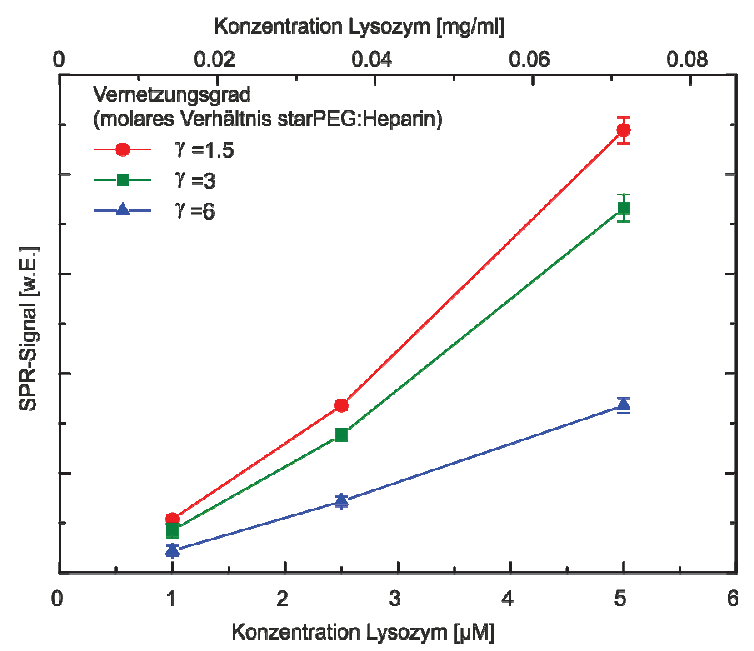

Bild 4: Verschiebung des Minimas bei den SPR-Messungen in Abhängigkeit der Lysozymkonzentration und des Vernetzungsgrades des Geles.

Damit konnte gezeigt werden, dass die Anbindung von Proteinen an die Hydrogele mittels Oberflächenplasmonresonanzspektroskopie detektiert werden kann.

\section{Elektrokinetische Messungen}

Die Grenzflächenleitfähigkeit wurde unter Verwendung der Mikrospaltzelle [4] über Strömungspotential- und Strömungsstrommessungen bestimmt. Diese hat in den durchgeführten Versuchsreihen mit zunehmender Beladung der Hydrogelfilme mit Lysozym infolge der damit einhergehenden Ladungskompensation deutlich abgenommen (Bild 5). Damit konnte nachgewiesen werden, dass die Grenzflächenleitfähigkeit ein geeigneter Parameter zur Detektion der Bindung von Biomolekülen in BiohybridHydrogelen ist.

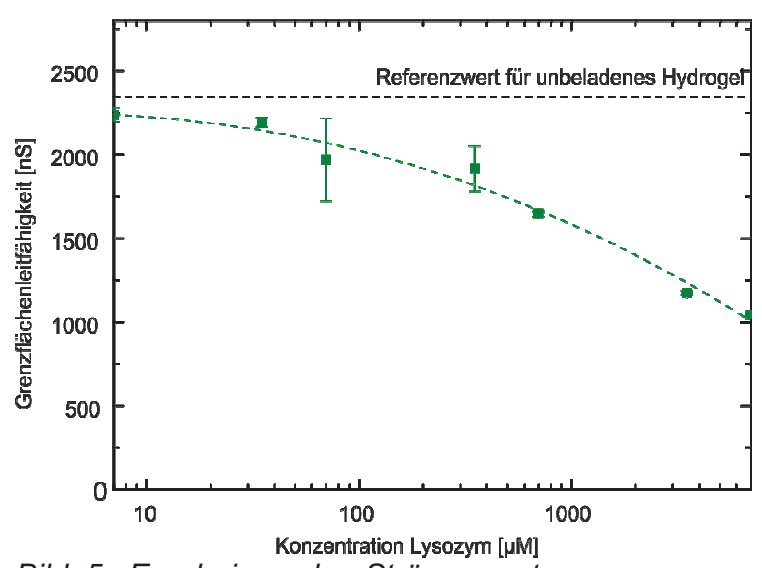

Bild 5: Ergebnisse der Strömungsstrommessungen an einem Hydrogel mit dem Vernetzungsgrad $\gamma=3$, welches mit unterschiedlichen Konzentrationen Lysozym beladen wurde.

\section{Zusammenfassung}

In der ersten Phase des Projektes konnten Biohybrid-Hydrogele aus starPEG und Heparin erfolgreich auf Sensorchips immobilisiert und 
erste Messungen zur Anbindung von Biomolekülen an die Gele durchgeführt werden.

Diese Arbeiten werden gegenwärtig auf Wachstumsfaktoren mit unterschiedlicher Affinität zu Heparin erweitert. Auf Basis der zu erwartenden Ergebnisse soll im weiteren Projektverlauf ein mikrofluidischer Sensor für die In-situ-Detektion von Bindungsereignissen in Biohybrid-Hydrogelen entwickelt werden.

\section{Danksagung}

Die Arbeit wird vom Sächsischen Ministerium für Wissenschaft und Kunst gefördert.

\section{Referenzen}

[1] U. Freudenberg, A. Hermann. P. B. Welzel, K. Stirl, S. C. Schwarz, M. Grimmer, A. Zieris, W. Panyanuwat, S. Zschoche, D. Meinhold, A. Storch, C. Werner, A star-PEG-heparin hydrogel platform to aid cell replacement therapies for neurodegenerative diseases, Biomaterials,30, 5049-5060, 2009; DOI:

10.1016/j.biomaterials.2009.06.002

[2] A. Kick, F. Götze, M. Mertig, Mikrokontaktdrucken zur Kontrolle von Flüssigkeitsmorphologien bei der Herstellung von DNA-Mikroarrays, 7. Deutsches BioSensor-Symposium 2011, 66-67, 2011

[3] J. Lyklema, Fundamentals of Interface and Colloid Science, Vol. II: Solid-Liquid Interfaces, Academic Press, London, 1995.

[4] R. Zimmermann, T. Osaki, R. Schweiß, C. Werner, Electrokinetic microslit experiments to analyse the charge formation at solid/liquid interfaces, Microfluidics Nanofluidics 2, 367-379, 2006; DOI: 10.1007/s10404-006-0087-6 